\title{
BMJ
}

\section{Patients' attitudes to the summary care record and HealthSpace: qualitative study}

\author{
Trisha Greenhalgh, professor ,' Gary W Wood, honorary senior research associate, ${ }^{1}$ Tanja Bratan, research \\ fellow , ${ }^{1}$ Katja Stramer, senior research fellow, ${ }^{1}$ Susan Hinder, freelance researcher ${ }^{2}$
}

${ }^{1}$ Department of Primary Care and Population Sciences, University College London, London N19 5LW ${ }^{2}$ RaFT Consulting, Clitheroe, Lancashire BB7 4BN

Correspondence to: T Greenhalgh p.greenhalgh@pcps.ucl.ac.uk

Cite this as: BMJ 2008;:elocator a114

doi:10.1136/bmj.a114

\section{ABSTRACT}

Objective To document the views of patients and the public towards the summary care record (SCR, a centrally stored medical record drawn from the general practice record) and HealthSpace (a personal health organiser accessible through the internet from which people can view their SCR), with a particular focus on those with low health literacy, potentially stigmatising conditions, or difficulties accessing health care.

Design 103 semistructured individual interviews and seven focus groups.

Setting Three early adopter primary care trusts in England where the SCR and HealthSpace are being piloted. All were in areas of relative socioeconomic deprivation.

Participants Individual participants were recruited from general practice surgeries, walk-in centres, out of hours centres, and accident and emergency departments. Participants in focus groups were recruited through voluntary sector organisations; they comprised advocates of vulnerable groups and advocates of people who speak limited English; people with HIV; users of mental health services; young adults; elderly people; and participants of a drug rehabilitation programme.

Methods Participants were asked if they had received information about the SCR and HealthSpace and about their views on shared electronic records in different circumstances.

Results Most people were not aware of the SCR or HealthSpace and did not recall receiving information about it. They saw both benefits and drawbacks to having an SCR and described a process of weighing the former against the latter when making their personal choice. Key factors influencing this choice included the nature of any illness (especially whether it was likely to lead to emergency care needs); past and present experience of healthcare and government surveillance; the person's level of engagement and health literacy; and their trust and confidence in the primary healthcare team and the wider NHS. Overall, people with stigmatising illness were more positive about the SCR than people who claimed to speak for "vulnerable groups." Misconceptions about the SCR were common, especially confusion about what data it contained and who would have access to it. Most people were not interested in recording their medical data or accessing their SCR via HealthSpace, but some saw the potential for this new technology to support self management and lay care for those with chronic illness. Conclusion Despite an extensive information programme in early adopter sites, the public remains unclear about current policy on shared electronic records, though most people view these as a positive development. The "implied consent" model for creating and accessing a person's SCR should be revisited, perhaps in favour of "consent to view" at the point of access.

\section{INTRODUCTION}

A large scale IT project is underway in England to place a summary version of the electronic patient record (the summary care record or SCR) on a central store, accessible on a secured extranet - known as N3-by a wide range of National Health Service (NHS) staff and made accessible to patients via the "HealthSpace" website. $^{1-3}$ Box 1 summarises details of the SCR. HealthSpace is a personal health organiser accessible through the internet on which people can store their medical details (such as blood pressure readings) and which also serves as an interface for NHS patients to view their SCR. For storing their own medical details, individuals must create a basic HealthSpace account; to view their SCR, they must create an advanced account.

Protagonists of shared electronic patient records anticipate several benefits. Clinical care, especially in the emergency setting, will (they believe) be better informed; fewer medical errors will occur; handovers between clinical teams will be smoother; people with limited English and those with low health literacy will receive as high a standard of care as everyone else; patients will be empowered; and as the efficiency of care rises, its costs will fall. ${ }^{45}$ Opponents have argued that the risks in terms of practicality, technical complexity, cost, and threats to confidentiality outweigh any potential clinical benefits. ${ }^{67}$ Some authors take a middle ground, arguing that projects like the SCR are neither assured of success nor doomed to failure but will depend crucially on such matters as effective business management, engagement of clinicians, and active participation of patients..$^{8-11}$

As part of a wider evaluation of the SCR and HealthSpace in early adopter primary care trusts, we sought to explore the attitudes of patients and the 
public to these new technologies, which had recently been introduced in their area.

\section{METHODS}

\section{Management and governance}

A research advisory group was set up with a lay chair and representatives of patients, clinicians, professional bodies (British Medical Association and Royal College of Nursing), and academic peers. Full details are given in the evaluation report. ${ }^{12}$

\section{Setting}

We carried out the study in three primary care trusts who were participating in the early adopter programme for the SCR. All had higher than average levels of socioeconomic deprivation and lower than average levels of limiting long term illness; ethnic mix was similar to the UK average.

\section{Sampling and recruitment}

Individual participants were recruited from general practices, out of hours centres, accident and emergency departments, and walk-in centres. Potential participants were generally approached by someone who was not a member of the research team (such as a bookingin clerk) and, if they were interested, were given a "plain English" invitation to participate, followed by a more detailed information sheet. As a requirement of the Research Ethics Committee, "special" (that is, shorter and simpler) invitation letters and information sheets had been prepared to be offered to those with cognitive difficulties or low health literacy, or both. Verbal

\section{Box 1 Key characteristics of the summary care record}

\section{The technology}

The summary care record (SCR) is a centrally stored summary of key medical details that is created from a person's existing NHS record (currently, the detailed record held by their general practitioner) and made available to NHS staff in emergency and unscheduled care situations (accident and emergency departments, general practice out of hours clinics, and walk-in centres). It is comparable with (but differs in important respects from) the emergency care summary in Scotland and the individual health record in Wales.

\section{What information does the SCR contain?}

Information held on the SCR is currently limited to current medication, allergies, and adverse reactions (the "phase 1 upload"), but a minimum clinical dataset (such as whether someone has diabetes-the "phase 2 upload") is being developed and added in selected sites.

\section{Security safeguards}

Extensive technical safeguards have been built into the SCR to prevent unauthorised access. Role based access controls restrict access to NHS staff with a legitimate relationship to the patient. Access by staff without such relationships are logged and audited; penalties for unauthorised access are severe and might include dismissal.

\section{The consent model}

The current consent model for the SCR is one of implied consent or "opt out" (that is, unless a person explicitly withdraws consent, an SCR will be created). Patients can choose one of three options: "don't store" (a blank SCR will be created; nothing will be uploaded beyond the demographic details that are already on the spine); "store and share" (a full SCR will be created); or "store but don't share" (a full SCR will be created but explicit consent must be obtained from the patient every time a health professional wants to access it). There will also be an option for a "virtual sealed envelope"-a "store but don't share" option applied to selective sensitive information. informed consent was obtained before we provided further details of the study. Participants were not required to give their names but were asked their age and occupation. To decide which set of paperwork to provide (standard or special), health literacy was estimated as follows:

- High: articulate, finds the invitation and background information sheet easy to read and understand, rapidly grasps explanations about the SCR, makes comments or asks questions that suggest good understanding

- Medium: able to read the information sheet without problems, seems to grasp explanations about the study and the SCR; researcher judges that standard invitation letter is likely to be understood

- Low: seems unable to read or understand the standard information sheet or to grasp basic issues about the SCR despite repeated explanations; researcher judges that the special invitation letter and information sheet should be offered.

Focus group participants were recruited from voluntary sector organisations and NHS interpreting and advocacy services (box 2). All participants provided written informed consent. In all groups except "advocates of vulnerable groups" (which was undertaken as a requirement of the ethics committee to identify potential access and consent issues for vulnerable participants), participants received a modest cash payment for expenses. Participants on the drug recovery programme received this in shopping vouchers.

\section{Individual interviews}

Individual interviews were intentionally brief (around five minutes) and were not recorded, so as not to put people off participating. We asked the following questions, adapted flexibly to fit with the person's responses:

- Do you know anything about electronic [computer] health records?

- Did you get a letter/have you heard about the SCR [explain if necessary]?

- Would you want an SCR? Why/why not?

- What would you see as the benefits of the SCR?

- What would you see as the disadvantages of the SCR?

- Any other concerns?

- Have you heard of HealthSpace? [explain if necessary]

- Would you want an advanced HealthSpace account to see a summary of your medical record? Why/why not?

We made contemporaneous notes on paper, writing down responses verbatim as much as was practical. Recruitment continued until saturation of themes was achieved.

\section{Focus groups}

Focus groups were held at community venues with which participants were already familiar. After 


\section{Box 2 Participants on focus groups}

\section{Advocates of vulnerable groups}

This group was initially convened as a requirement of the research ethics committee to identify key issues around vulnerable groups before such groups were approached directly. We approached public and voluntary sector organisations, and participants represented NHS patient involvement groups (two people); local councils (with responsibility for homeless and refugees); gay and lesbian groups; people with learning difficulties; mental health services users; HIV/AIDS groups (two people); an advice centre foryoung people; and a charity supporting victims of domestic violence. Representatives from organisations for elderly people and ethnic minorities were invited and showed interest but were unable to attend because of logistical problems on the day.

\section{Advocates of people with limited spoken English}

This group was convened from people interested in supporting others in their community who sought to access health care and other services. All but one were first generation immigrants who had not learnt English before arriving in the UK. The group comprised two lay people, two facilitators for the expert patient programme, three lay interpreters (who, for example, interpreted regularly for their own family members in healthcare consultations), and three professional bilingual health advocates. They represented seven different ethnic groups and six countries of origin in South Asia, Africa, and eastern Europe. They had lived in the UK for between four and 30 years.

\section{People with HIV}

This group was held in a voluntary sector support centre for those with HIV. The centre had a large social space, a kitchen, and a playroom for children. Volunteers gave general support and advice on benefits and accessing care. Participants comprised five white men (three of whom volunteered that they were gay), five African women (two with babies), and one African man. One person was a health professional who had contracted HIV through occupational contact.

\section{Users of mental health services}

This group was convened in association with MIND (a mental health association) and held at the local MIND offices. All participants were users of mental health services; several also had a relative with a mental health condition. We did not ask about participants' mental health problems but conditions volunteered included bipolar disorder, depression, "feeling suicidal," "being sectioned," "needing counselling," "finding things scary," "finding it extremely difficult to communicate with people," "not always being rational," and "personality problem."

\section{Older people}

This group was convened through a lunch club for older people in a suburban area. Participants regularly attended the lunch club, which meets once a week, as well as arranging holidays and day trips. The focus group was run before the regular meeting in its usual venue-a sports and recreation club.

\section{Young people}

This group was held at the Brook Advisory Centre, a voluntary sector organisation for sexual health advice. Participants were recruited via the Brook reception and support staff; they comprised both Brook clients and their social contacts.

\section{People on a drug recovery programme}

This group was convened in association with Turning Point in the west Midlands and held at one of its centres in an inner city area. All participants were attending a structured recovery programme. The focus group was run during a session reserved for outside speakers on the programme, and it was made clear in advance that the session would involve voluntary participation in research. No questions were asked regarding the participants' background or patterns of drug use. Some participants, however, volunteered information that indicated injecting drug use. stories. Box 3 gives an indicative list of discussion topics. Focus group discussions were transcribed and independently checked; contemporaneous notes were also made during the groups.

\section{Data analysis}

We conducted an initial content analysis of free text responses in the individual interviews (by counting the number of people who mentioned a particular topic such as security). ${ }^{13}$ In a more detailed qualitative analysis, three researchers independently coded the write-ups of individual interviews and focus group transcripts. We held a series of discussion meetings to refine these coding categories and developed a shared analytic framework based on the method of Ritchie and Spencer, ${ }^{14}$ which we transferred to an Excel spreadsheet for more detailed coding and analysis. Disagreements between our individual interpretations were resolved by discussion.

\section{RESULTS}

\section{Demographic characteristics}

Table 1 (individuals) and table 2 (focus groups) show the characteristics of the sample. Overall, $41 \%$ of our sample were men; 83\% were white, $8 \%$ Asian, 6\% African, and $3 \%$ mixed race. Of those who chose to give their occupation, most described white collar, manual, or homemaker jobs, and $6 \%$ were unemployed; we estimated $20 \%$ of the sample to have high health literacy, 45\% medium, and 38\% low. People attending the walk-in centres seemed to represent a particularly deprived group (typically, young mothers seeking advice on a minor health problem in a toddler) and accounted for a high proportion of the participants with low health literacy. About a third of individuals approached, and a similar proportion of people invited to join focus groups, chose not to participate in the study.

\section{Awareness of the SCR and HealthSpace}

Table 3 summarises the views of individual participants. Official statistics suggest that by the date of the interview, around $95 \%$ of the population in our sample area had been sent a letter informing them that the SCR was being introduced in their area. Only about one in seven recalled receiving this letter. Overall, 29\% were aware of the SCR (some via the media or their general practitioner) and $8 \%$ were aware of HealthSpace. Awareness of the SCR was higher in those we had classified as having high health literacy (one in two aware) than those with medium or low health literacy (one in four aware). Many believed (wrongly) that electronic records were already shared between health professionals either locally or nationally.

explaining the study, the researcher asked if anyone had heard of the SCR and HealthSpace, explained these if necessary, and invited comments. Differences in views were explicitly explored by asking participants to comment on what others said about particular

\section{"Benefits" v "drawbacks"-a personal equation}

Most people were positive about the SCR and happy that if they did nothing, one would be created for them. But few were unequivocally in favour of the idea. Rather, people described a process of weighing 


\section{Box 3 Indicative list of topics for focus group discussions}

This list was adapted flexibly in response to emerging themes.

- Explain purpose of study and gain verbal consent and commitment to confidentiality on tape from all participants

- Ask if anyone knows about electronic records and if they'd like to tell the others about them

- Follow-up on any interesting stories raised spontaneously about electronic records (for example, What do others think about that story? Has anyone had a different experience?)

- Ask if anyone has been approached about the SCR or if they know what it is

- Follow up on any stories raised by participants about the SCR

- Ask about other experiences with large scale IT systems-for example, Does anyone do their shopping on the internet or use internet banking? What do you think of those services? Why do you (or don't you) trust them? What do you think about their security level? If you don't think about that, why not-what makes you trust them?

- Introduce vignette style prompt-for example, Let me tell you about Fred. Fred is HIV positive and he also has diabetes. He goes to the hospital for some of his diabetes care but he also sees his GP. He quite likes the idea of the SCR for his diabetes care but he has concerns about his HIV status being seen by people who don't really need to know that information. What do you think is going through Fred's mind when he gets the letter about the SCR? What do you think he'll decide to do and why? Fred gets told that there is a "virtual sealed envelope" for private information, and that he can ask to have his HIV status put in that part of the electronic record if he wants. What do you think Fred would think of the virtual sealed envelope?

- Ask what sort of person would NOT want to have their medical record stored electronically in the form of an SCR? What would that person's reservations be? What might change their mind?

- (If technical facilities available) show training DVD of SCR and ask what people think of it

perceived benefits (box 4) against drawbacks (box 5) for them personally and, when relevant, their dependent relatives. The balance between benefits and drawbacks was different in different situations (as the parent of a child with a behaviour disorder put it, "records are very delicate things"). We have analysed the benefit-drawback equation below in terms of a series of tensions.

The most commonly cited factor influencing the decision to have an SCR was personal experience. People who had had an adverse drug reaction, an episode of loss of consciousness, lost medical records, or a "near miss" medical error, and those with serious or complex health problems (especially those with multiple comorbidity) tended to view the SCR positively ("I suffer from these mini strokes, if I'm away on holiday and I had one of them, they would know exactly," older person in focus group). Those who had been the victim of mistaken identity (in the NHS or outside it), an incorrect medical diagnosis, or identity fraud (such as stolen credit card) tended to be opposed to it. Those who lacked relevant personal experience were often undecided about, or disinterested in, the SCR.

For most people, the personal risk-benefit equation came out in favour of having a SCR but against having a HealthSpace account. Many were unsure of the purpose of HealthSpace, describing it as "pointless," "irrelevant," and not fit for purpose ("I would just rather write it down in the diary or just hide it underneath my bed or something," young person in focus group). They also saw HealthSpace as a potential weakness in the system in terms of security. Several said that they preferred to discuss their health issues with their own general practitioner or nurse (or in one case, a favourite receptionist). We interviewed one person

Table 1| Demographic characteristics of sample for individual interviews in 103 participants

\begin{tabular}{|c|c|c|c|c|c|}
\hline & \multicolumn{4}{|c|}{ Source of recruitment } & \multirow[b]{2}{*}{ Total } \\
\hline & GP surgeries & Walk-in centres & A\&E departments & $\begin{array}{l}\text { Out of hours } \\
\text { centres }\end{array}$ & \\
\hline Median (range) age (years) & $45(25-75)$ & $28(16-78)$ & $50(21-59)$ & $36(34-53)$ & $35(16-78)$ \\
\hline Men & 22 & 14 & 7 & 2 & 45 \\
\hline Women & 22 & 32 & 3 & 1 & 58 \\
\hline \multicolumn{6}{|l|}{ Ethnicity: } \\
\hline White & 39 & 43 & 10 & 3 & 95 \\
\hline South Asian & 4 & 1 & 0 & 0 & 5 \\
\hline African & 1 & 2 & 0 & 0 & 3 \\
\hline \multicolumn{6}{|l|}{ Occupation: } \\
\hline Professional or managerial & 2 & 5 & 5 & 0 & 12 \\
\hline "White collar" & 0 & 11 & 2 & 0 & 13 \\
\hline Manual & 5 & 8 & 2 & 1 & 16 \\
\hline Unemployed & 2 & 4 & 0 & 2 & 8 \\
\hline Housewife & 16 & 12 & 0 & 0 & 28 \\
\hline Student & 0 & 5 & 0 & 0 & 5 \\
\hline Missing/did not want to say & 19 & 1 & 1 & 0 & 21 \\
\hline \multicolumn{6}{|l|}{ Estimated health literacy: } \\
\hline High & 11 & 3 & 6 & 0 & 20 \\
\hline Medium & 27 & 14 & 2 & 2 & 45 \\
\hline Low & 6 & 29 & 2 & 1 & 38 \\
\hline Total & 44 & 46 & 10 & 3 & 103 \\
\hline
\end{tabular}

A\&E=accident and emergency. 
Table 2 | Demographic characteristics of sample for focus groups

\begin{tabular}{|c|c|c|c|c|c|c|c|c|}
\hline & $\begin{array}{c}\text { Advocates of } \\
\text { vulnerable groups }\end{array}$ & $\begin{array}{l}\text { Advocates of limited } \\
\text { English speakers }\end{array}$ & $\begin{array}{c}\text { HIV } \\
\text { positive }\end{array}$ & $\begin{array}{l}\text { Mental health } \\
\text { service users }\end{array}$ & $\begin{array}{l}\text { Older } \\
\text { people }\end{array}$ & $\begin{array}{l}\text { Young } \\
\text { people }\end{array}$ & $\begin{array}{l}\text { People on drug } \\
\text { recovery programme }\end{array}$ & Total \\
\hline Age range* & $30-55$ & $30-60$ & $25-50$ & $18-60$ & $69-84$ & $17-21$ & $27-45$ & $17-84$ \\
\hline Men & 3 & 0 & 6 & 2 & 5 & 4 & 6 & 26 \\
\hline Women & 7 & 10 & 5 & 5 & 7 & 4 & 3 & 41 \\
\hline \multicolumn{9}{|l|}{ Ethnicity: } \\
\hline White & 10 & 1 & 5 & 7 & 12 & 4 & 7 & 46 \\
\hline South Asian & 0 & 7 & 0 & 0 & 0 & 0 & 1 & 8 \\
\hline African & 0 & 2 & 6 & 0 & 0 & 0 & 0 & 8 \\
\hline Mixed race & 0 & 0 & 0 & 0 & 0 & 4 & 1 & 5 \\
\hline \multicolumn{9}{|l|}{ Occupation: } \\
\hline $\begin{array}{r}\text { Professional/ } \\
\text { senior manager }\end{array}$ & 10 & 0 & 1 & 0 & 0 & 0 & 0 & 11 \\
\hline "White collar" & 0 & 8 & 1 & 0 & 0 & 0 & 0 & 9 \\
\hline Manual & 0 & 0 & 0 & 0 & 0 & 0 & 0 & 0 \\
\hline Unemployed & 0 & 0 & 4 & 0 & 0 & 0 & 0 & 4 \\
\hline Housewife & 0 & 2 & 3 & 0 & 0 & 0 & 0 & 5 \\
\hline Student & 0 & 0 & 0 & 1 & 0 & 0 & 0 & 5 \\
\hline $\begin{array}{l}\text { Missing/did not } \\
\text { want to say }\end{array}$ & 0 & 0 & 2 & 6 & 12 & 8 & 9 & 33 \\
\hline \multicolumn{9}{|c|}{ Estimated health literacy: } \\
\hline High & 10 & 8 & 3 & 2 & 4 & 0 & 0 & 28 \\
\hline Medium & 0 & 2 & 4 & 3 & 8 & 8 & 4 & 29 \\
\hline Low & 0 & 0 & 4 & 2 & 0 & 0 & 5 & 11 \\
\hline Total & 10 & 10 & 11 & 7 & 12 & 8 & 9 & 67 \\
\hline
\end{tabular}

*Ages estimated when not stated.

who had signed up for a HealthSpace account, accessed their record, and was now trying to de-register because he had found it far less comprehensive and useful than he had expected. A small but important minority, however, saw potential benefit of HealthSpace for keeping track of their own or a relative's chronic illness.

Tension 1: Reducing or exacerbating the impact of stigmatising illness

Many participants (including advocates who claimed to speak for "vulnerable groups") assumed that someone with a potentially stigmatising condition would not want an SCR. But most people who actually had a serious long term condition thought that the risk of disclosure to a third party was outweighed by the benefits of having an accessible record. Two individual interviewees spontaneously disclosed their own epilepsy, for example, as a reason why they were strongly in favour of the SCR in case they collapsed unconscious.

The idea of a "virtual sealed envelope" (see box 1) for sensitive information was viewed positively by some participants in the focus group (who cited sexually transmitted diseases or termination of pregnancy as examples of things people would want to keep private) but negatively by others. Mental health service users and those on a drug rehabilitation programme, for example, expressed concern that doctors might get a distorted picture of their health needs if key information was missing, or assume a more stigmatising diagnosis than the one that was "sealed" ("they might think you're a psycho"... "or a fraggle [sex offender]," participants in drug rehabilitation focus group). Some but not all people with HIV (especially onewho had contracted it through occupational exposure) thought that their status was nothing to be ashamed of and that seeking to hide it in a "sealed envelope" would add to the stigma.

Tension 2: Increasing or decreasing access to health care for vulnerable groups

Advocates of people with limited spoken English said that the SCR might make it easier for disempowered minority ethnic groups to access and register with a new general practitioner as some general practitioners were (allegedly) known to use lack of the proper paperwork (such as proof of identity) as a reason not to register a patient. They also thought that the presence of an SCR would enable an unscrupulous general practitioner to preview someone's record before accepting them and selectively turn away those with complex, expensive to treat, or poorly controlled diseases. Participants in several focus groups suggested that once the SCR was introduced nationally, illegal immigrants might "go underground" rather than seek health care.

Tension 3: Increasing or decreasing the quality and efficiency of care

Participants with chronic illness described frustrating experiences in hospital outpatient departments when their paper records had been unavailable and they had had to have repeat blood tests, $\mathrm{x}$ rays, or come back on another day, and anticipated that the SCR would make 
Table 3 | Awareness of, and decisions about, the SCR and HealthSpace in NHS service users $(n=103)$

\begin{tabular}{|c|c|c|c|c|}
\hline & \multicolumn{3}{|c|}{ Estimated health literacy } & \multirow[b]{2}{*}{ Total } \\
\hline & Low $(n=38)$ & Medium $(n=45)$ & High $(n=20)$ & \\
\hline \multicolumn{5}{|l|}{ Awareness of SCR: } \\
\hline Received letter from GP and/or PCT & 4 & 5 & 5 & 14 \\
\hline Saw leaflet in GP surgery: & 0 & 3 & 0 & 3 \\
\hline $\begin{array}{l}\text { Aware through mass media } \\
\text { (newspaper, radio) }\end{array}$ & 3 & 1 & 2 & 7 \\
\hline Other (such as health professional) & $1^{\star}$ & 1 & 1 & 4 \\
\hline Did not state how became aware & 0 & 2 & 1 & 3 \\
\hline Total No aware of SCR & 9 & 12 & 9 & 30 \\
\hline \multicolumn{5}{|c|}{ Of those $(n=30)$ who said they had received letter or were otherwise aware of SCR: } \\
\hline $\begin{array}{l}\text { Took no action as happy to have an } \\
\text { SCR }\end{array}$ & 7 & 7 & 7 & 21 \\
\hline $\begin{array}{l}\text { Did not want an SCR so actively opted } \\
\text { out }\end{array}$ & 0 & 1 & 0 & 1 \\
\hline $\begin{array}{l}\text { Still considering whether to have an } \\
\text { SCR }\end{array}$ & 0 & 1 & 2 & 3 \\
\hline $\begin{array}{l}\text { Put letter or leaflet aside to think about } \\
\text { later }\end{array}$ & 1 & 1 & 0 & 2 \\
\hline Binned letter as junk & 1 & 2 & 0 & 3 \\
\hline \multicolumn{5}{|l|}{ Decision about SCR: } \\
\hline Yes would like one & 18 & 31 & 15 & 64 \\
\hline Not sure/haven't made mind up & 2 & 3 & 3 & 8 \\
\hline Don't care/not bothered & 8 & 0 & 0 & 8 \\
\hline No don't want one & 5 & 3 & 0 & 8 \\
\hline Changed mind during interview & 2 & 0 & 0 & 2 \\
\hline Missing data & 4 & 8 & 2 & 14 \\
\hline \multicolumn{5}{|l|}{ Awareness of HealthSpace: } \\
\hline Had previously heard of HealthSpace & 0 & 6 & 2 & 8 \\
\hline $\begin{array}{l}\text { Had not previously heard of } \\
\text { HealthSpace }\end{array}$ & 34 & 35 & 15 & 83 \\
\hline Missing data & 4 & 3 & 3 & 10 \\
\hline \multicolumn{5}{|l|}{ Decision about HealthSPace: } \\
\hline Yes would like HealthSpace account & 3 & 11 & 9 & 23 \\
\hline Not sure/haven't made mind up & 1 & 5 & 4 & 10 \\
\hline No, definitely don't want one & 31 & 25 & 6 & 62 \\
\hline Changed mind during interview & 1 & 0 & 0 & 1 \\
\hline Missing data & 2 & 4 & 1 & 7 \\
\hline Men & 11 & 20 & 11 & 45 \\
\hline Women & 24 & 25 & 9 & 58 \\
\hline Median (range) age (years) & $40(16-75)$ & $29(16-78)$ & $35(24-70)$ & $35(16-78)$ \\
\hline
\end{tabular}

such experiences a thing of the past. Many assumed that their SCR would necessarily be complete, accurate, and universally accessible. They saw a link between these aspects of the record and quality of care ("I would like to go to somewhere and be treated properly, with all my record," participant in HIV focus group). But others rejected a view of the SCR as a "smart machine," protecting against the impact of staff error. They thought the SCR was likely to require sophisticated skills and consistent practices and that the quality of data on the SCR would only be as good as the data quality standards and practices that support its creation and maintenance. If the NHS could not get "simple" paper records right (they reasoned), difficulties were even more likely with shared electronic records.
Tension 4: Making care more objective-or entrenching prejudice

Some people believed that the SCR would provide dispassionate information to support "objective and "professional" care. Mental health service users, for example, pointed out that their condition (and their ability to give a credible account of themselves) fluctuated; having it documented on an SCR might enable them to be taken seriously in an emergency rather than turned away as "stroppy." Others, however, were concerned that a diagnosis on the SCR might lend false objectivity to impressionistic or one off assessments, especially of a person's mental state, thereby colouring the judgment of others in the future. 
Box 4 Benefits of the SCR perceived by service users

Individual interviews $(\mathrm{n}=103)$

Commonly mentioned (by more than $10 \%$ of the sample)

- SCR is a "good thing" (unspecified or "the more information the better")

- Having medical details safely and consistently in one place, especially in emergency situations or chronic/ complex illness

- Makes care easier/more efficient/saves time/helps you fill out other forms

- Not having to answer questions, fill out forms, or remember what medication you are on

Less commonly mentioned (by less than $10 \%$ of the sample)

- Stops people giving you the wrong medication or medication that you are allergic to

- Medical record available when not near own general practitioner/can move house without changing general practitioner*

- Could prevent a recurrence of a previous bad experience (lost medical record, duplicate blood test, bad allergic reaction, collapse)

- Can print off for own records or to take to another healthcare professional

- Provides evidence about a problem that patient knows they have but which health professionals might doubt

- Stops people lying (because it provides an "objective" version of reality)

- Useful for deaf people

Additional themes raised in focus groups (seven groups, 67 participants in total)

- The SCR could be printed out and taken to another clinician for a second opinion

- New immigrants might change general practitioner often and often have particular problems articulating key aspects of their medical record (some of which might be traumatic). The SCR will help continuity of care in this group

- Potential research uses of aggregated data from SCR

- If someone has an SCR a general practitioner would not be able to refuse treatment pending arrival of records

- Useful for elderly people who might be forgetful but on lots of different tablets

- "To put my side of the story"*Two people independently described the SCR as a "fantastic" idea because (in their view) it allowed them to remain registered with a GP in a different part of the country after moving house.

Tension 5: Increasing or decreasing patient empowerment Some participants said they would like to view their SCR to see what the doctor had written about them, and believed (incorrectly) that the SCR would contain explanations and clarifications of what had been said in a primary care or outpatient consultation or why they had been sent for a test. Advocates of those with limited spoken English suggested that such people valued the written word (particularly numerical data) over oral explanations as these would be more readily assimilated by someone with limited English.

One of the commonest perceived benefits of the SCR, however, was its potential to save having to fill out forms or remember what medication one was taking. Many participants whom we judged to have low health literacy gave "not bothered" or "don't care" responses to the question "Would you like to have an SCR?" often citing limited capacity to understand ("I can't get my head round it"). As box 6 shows, the overwhelming reason for people in this sample not wanting a HealthSpace account was uninterest in their own health record and, in a few cases, an active distaste for seeing information about their illnesses ("I wouldn't want to look nothing up on myself, like"). Older people found the idea of accessing their personal health data amusing ("You'll become a hypochondriac!" older person in focus group).

Tension 6: Increasing or decreasing clinician-patient trust A positive attitude towards the SCR was often linked to implied trust in the honesty and motives of NHS staff ("[they will] just see what's wrong with you. It's not [as] if they're up to jack [rob] you or nothing," individual participant). In situations where trust in the clinician was low, the SCR and HealthSpace were seen as potentially able to legitimisethe patient's account of reality. For example, some patients hoped to use these technologies to "prove" that they had genuinely been ill on a previous occasion or were really taking the tablets they claimed to be taking. Where trust was high, access to one's medical record was viewed as unnecessary and even undesirable. At one general practice, for example, there was strong resistance to the idea of HealthSpace as patients seemed to think that this would undermine their good relationship with surgery staff ("It's a terrific surgery so there's no need for something like HealthSpace," individual participant).

Some participants (especially mental health service users) thought that NHS staff had dismissive attitudes towards them, and some had little confidence that the SCR would be used as intended. Trust (or lack of trust) in a member of NHS staff seemed to be a feature of the relationship with a particular individual rather than of that person's formal role or job status ("He could be a snidey little GP," young person in focus group) and seemed to be closely linked to continuity of care ('I'm perfectly happy for anybody at my doctor's to look at my records because I know everybody at my doctors. I'm more happy for them to have my files, but anybody else, no," participant in drug rehabilitation focus group). Most participants, particularly those with chronic illnesses, wanted to have an SCR but also wanted to control who had access to it at the point of care.

Tension 7: The state as protector or exploiter of the citizen's data

People were relieved to hear about the extensive technical and operational security measures associated 
with the SCR (which include secure extranet access, password and role based access controls, and penalties for attempting to access a patient's record inappropriately). ${ }^{12}$ While not a single participant thought that these measures would guarantee the security of their data, most thought that the small risk of identity fraud, disclosure, or blackmail was worth taking. They contrasted personal health information (seen as a low security risk) with their bank details (much higher risk), and some people with serious illness joked that nobody would want to steal their identity.

Around one in 12 people thought that the SCR was a bad idea "on principle," viewed the intention to create one as an infringement of their rights, and drew explicit parallels with government plans to introduce identity cards and the clamp down on social security fraud (which some saw as covertly linked to the SCR). Some were concerned that once consent for an SCR had been given, pressure would build from a host of public and private sector organisations to access the data, and the unscrupulous government would soon be tempted to make money from a range of secondary uses.

\section{DISCUSSION}

Our study, published just as the Department of Health plans to extend the SCR programme from a few early adopter sites to much of the country, has shown that a person's decision to have (or not to have) a summary care record (SCR) is both individual and complex. The benefits of the SCR (especially the availability of medical information in an emergency situation) must be weighed against its drawbacks (such as the risk of security breaches, human error, the potential stigma of disclosure, or a label that becomes a self fulfilling prophecy) in a way that addresses personal priorities and context. Key mediating factors include the nature of the illness (especially whether it is likely to lead to emergency care needs when the individual is unable to communicate); the person's past and present experience of both healthcare and government surveillance; their level of engagement and health literacy; and their level of trust and confidence in the primary healthcare team, the NHS, and the government.

\section{The importance of sampling frame}

While there is a substantial body of literature on what patients think about access to electronic records held by their general practitioner, ${ }^{1516}$ and while doctors have published their own views on the ethics and practicality of the $\mathrm{SCR},{ }^{6-17}$ previous studies on patients' attitudes to internet based records have been limited. Pyper and colleagues showed that in a north Oxford population, accessing an online medical record from their general practitioner's surgery was feasible and acceptable to those patients who accepted the invitation. ${ }^{18}$ In that study, patients understood most of the entries; they had some concerns about security and confidentiality; and while many found errors, most of them were not medically important. Levels of health literacy and engagement in this sample, however, are

\section{Box 5 Drawbacks of the SCR perceived by service users}

Individual interviews ( $\mathrm{n}=103)$

- Malicious or inappropriate access

Hackers/fraudsters

Benefits agency

Employers/insurance companies/credit control agencies

Local NHS colleagues (unauthorised access to records of NHS staff)

Parents (in relation to pregnancy test or termination of pregnancy)

"The general public"

Receptionists

"Foreigners"

- Security breaches

Technical error (includes power cuts, system breakdown)

Human error or not enough people to run the system

- SCR a "bad thing" (unspecified or all computers are bad)

- Stigma/labelling (such as, depression, counselling, sexual infections, child with ADHD (attention-deficit/ hyperactivity disorder))

- Waste of money

- People won't understand their choices/too complicated

- NHS would need to provide more computers-for example, in operating theatres

- Mistaken identity (for example, similar name)

- If inaccurate, could cause more harm than good

Additional themes raised in focus groups (seven groups, 67 participants in total)

- Allows general practitioners to turn away patients who seek to register with them but might be expensive to treat

- Government would sell data to private companies

- Staff "incompetence" is likely to exacerbate problems if the SCR is introduced as more can go wrong

- An accurate and complete SCR depends on data quality standards and practices

- People with sexually transmitted infections might be open to blackmail as information indicating an affair could be passed to a spouse

- Discriminates against those who have chosen not to register with a general practitioner

- Family members could learn of a drug addiction

unlikely to be representative of the wider UK population. A pilot project on internet based medical records in Hampshire invited people to comment on their experience on a website, but only 20 of 1300 people who accessed the site completed a questionnaire, and while several of these had strong views, it is relevant that only a tiny fraction of people chose to comment. ${ }^{19}$

In contrast to these earlier studies, our own sample broadly reflected the ethnic mix of the general population and was purposely skewed towards lower sociodemographic groups, those with lower health 
literacy, and those with high health and social care needs. While the articulate "empowered" middle classes are under-represented in our sample, they are also under-represented in the section of the population who seek health care in emergency and unscheduled care situations (the main group for whom the SCR was designed $\left.^{11}\right)$.

\section{Health literacy}

Our approach to classifying people as having "low health literacy" (on the basis of whether we judged the participant to require an explanation of our research in "simple" English) was unsophisticated, pragmatic, and initially oriented towards our own consent procedures. Nevertheless, our classification revealed some important patterns in the data that we believe should be explored further. Our finding that the SCR is seen as a good thing because it reduces personal responsibility for health, links not with the assumed "empowerment" agenda that was said to underpin the introduction of the SCR and HealthSpace ${ }^{11}$ but with the "lack of engagement" agenda which Sir Derek Wanless warned could potentially undermine the success of numerous public health initiatives in the UK. ${ }^{20}$ In participants with low health literacy, lack of interest in one's own health seemed to be the key moderating factor that explained the mismatch between the decision to have an SCR (almost all "yes" or "don't care") and the decision to have a HealthSpace account (almost all "no"), and supports a recent finding by Hibbard et al that the "empowerment" agenda requires cognitive skills that not all citizens possess. ${ }^{21}$ This finding is preliminary, however, and should be explored further.

\section{Implications for the consent model}

Two key findings from this study - that many people fail to engage with the SCR until they have a relevant personal experience, and that trust is a feature of the interpersonal relationship rather than associated with particular healthcare roles-suggest that seeking a patient's consent to view their record should occur as far as possible at the point of access in an unscheduled care setting. A "consent to view" model, which is already in place for the emergency care summary in Scotland, might be both more pragmatic and more ethically justifiable than the current model of implied consent to upload, after which the record can be accessed by anyone who can claim a "legitimate relationship" with the patient.

We found several misconceptions about the SCR and HealthSpace (table 4). Importantly, many people do not understand the difference between the PDS (personal demographics service - a demographic database on which all NHS patients are listed), the electronic record held by the general practitioner, and the SCR. They have limited understanding of what data are currently shared or what technical and access control measures are in place to protect their data. These findings align with those of other surveys ${ }^{22}$ and are unsurprising given the inherent complexity and rapid development of electronic records in the NHS. In a world in which health care is supported by technologies that are beyond the awareness of most

\begin{tabular}{|c|c|c|}
\hline & Misconception & Comment \\
\hline 1 & $\begin{array}{l}\text { Doctors and nurses already have the ability to access a patient's full medical record } \\
\text { wherever they are in the NHS }\end{array}$ & This facility does not exist except on a limited local basis in some areas \\
\hline 2 & $\begin{array}{l}\text { The SCR and HealthSpace will contain detailed medical information that will (for example) } \\
\text { enable a person to clarify what the GP said in the last consultation or why a particular test } \\
\text { was ordered }\end{array}$ & $\begin{array}{l}\text { The SCR will focus mainly on current medication, allergies, and adverse reactions. As } \\
\text { currently planned, it will contain only brief medical details }\end{array}$ \\
\hline 3 & $\begin{array}{l}\text { If an SCR is created, it will contain an accurate and complete account of current illness and } \\
\text { will always be accessible to healthcare staff, making lost records or missing data a thing } \\
\text { of the past }\end{array}$ & $\begin{array}{l}\text { The SCR is unlikely to be universally accessible because of technical and operational } \\
\text { hurdles. Its future accuracy and completeness are unknown }\end{array}$ \\
\hline 4 & $\begin{array}{l}\text { "Opting out" of the SCR means that a person will no longer be registered on the personal } \\
\text { demographic service (PDS or "the Spine"). Hence, opting out will protect against identity } \\
\text { fraud }\end{array}$ & $\begin{array}{l}\text { All NHS patients will be registered on the PDS. Identity fraud (while highly unlikely) is } \\
\text { therefore a theoretical possibility even for patients who have opted out of the SCR }\end{array}$ \\
\hline 5 & $\begin{array}{l}\text { The SCR will be cross-linked to the social security system, making it possible for the } \\
\text { government to check up on sickness and incapacity benefit claims }\end{array}$ & No such plans have been announced \\
\hline 6 & $\begin{array}{l}\text { The SCR will enable people to remain registered with a GP even when they move out of the } \\
\text { area }\end{array}$ & A patient who moves out of area will still be expected to register with a new GP \\
\hline 7 & $\begin{array}{l}\text { An advanced HealthSpace account will allow a patient access to their detailed medical } \\
\text { record }\end{array}$ & HealthSpace allows access only to the SCR, not the detailed medical record \\
\hline 8 & $\begin{array}{l}\text { The SCR will contain details of sexually transmitted infections (STIs) and/or a person's } \\
\text { sexual orientation }\end{array}$ & $\begin{array}{l}\text { Information on sexual orientation is not part of the minimum dataset; STIs treated at } \\
\text { specialist clinics will not be on the GP record, and STIs treated by the GP would not normally } \\
\text { be part of the minimum dataset }\end{array}$ \\
\hline 9 & $\begin{array}{l}\text { The SCR will improve access to GPs for vulnerable groups because proof of identity and } \\
\text { current address will no longer be needed to register }\end{array}$ & The SCR will not change the requirements for registering with a GP \\
\hline 10 & $\begin{array}{l}\text { The SCR will allow the patient to legitimise an account of illness (for example, that they } \\
\text { were genuinely sick on a particular day in the past) }\end{array}$ & $\begin{array}{l}\text { The SCR is unlikely to contain sufficient detail to adjudicate in contested accounts of } \\
\text { illness }\end{array}$ \\
\hline 11 & The SCR could be easily hacked into & $\begin{array}{l}\text { Extensive technical security measures and access controls are in place, making hacking } \\
\text { unlikely though not impossible }\end{array}$ \\
\hline 12 & Patients will be able to make corrections to their medical record directly via HealthSpace & As currently planned, corrections will be possible only indirectly \\
\hline
\end{tabular}




\section{Box 6 Attitudes of service users to HealthSpace}

\section{Individual interviews $(\mathrm{n}=103)$}

Would not want any kind of HealthSpace account (total $n=62$ )

- Not interested, wouldn't want to see own record $(n=44)$

- Worried about security $(n=9)$

- Prefer to discuss my health with my general practitioner or other practice staff $(n=8)$

- Don't use the internet for anything/haven't got a computer/"I'm old fashioned" (n=7)

- Pointless, wouldn't tell me anything I don't already know $(n=6)$

- Registration process too much hassle $(n=4)$

- Worried about a family member or partner seeing $(n=3)$

- A printout of my general practice record would give all the information needed $(n=3)$

- "No time to mess around" $(n=1)$

Yes, would like some sort of HealthSpace account (total $n=23$ )

- Sounds like a good idea/sign of progress in the NHS $(n=5)$

- Would like HealthSpace to keep track of child or elderly parent's illnesses $(n=5)$

- Would like to see HealthSpace once, just to have a look at what is there $(n=4)$

- Have a lot of health problems myself, would like to keep track of them $(n=4)$

- Enjoy using computers/interested in playing with the technology $(n=2)$

- See my [child's] x ray pictures $(n=1)$

Undecided (total) 10

- Haven't thought about it, but haven't ruled it out $(n=6)$

- Might want it but it's not a priority ( $n=4)$

- Ambivalent (because of security worries) $(n=2)$

- Unable to understand explanation of what HealthSpace is $(n=2)$

Additional themes raised in focus groups (seven groups, 67 participants)

- Creates a route for hackers to access the SCR

- Not fit for purpose-could just as easily write personal health data down in a book or keep a file on a personal computer

- A person's medical record could be accessed by a partner without their full consent if they were in a coercive domestic relationship

- Registration process is complex and requires high IT literacy; this will discriminate against people with low literacy and those who are dyslexic

- Registration process requires a consistent date of birth, consistently spelt surname, and three pieces of identification—for example, utility bill, driving licence; refugees and asylum seekers might not have these documents and might use different spellings of their surname.

\section{WHAT IS ALREADY KNOWN ON THIS TOPIC}

The English Department of Health is about to extend a programme to create an electronic summary of medical details, accessible to NHS staff via the internet, for every NHS patient who does not opt out

Previous research in relatively affluent areas suggested that patients are willing and able to engage with decisions about their electronic medical record

\section{WHAT THIS STUDY ADDS}

Levels of awareness of, and knowledge about, the summary care record (SCR) and HealthSpace (to enable records to be viewed over the internet), especially among those with low health literacy, are currently low in early adopter sites, despite an extensive public information programme

People's attitudes to these new technologies vary but most are positive towards the SCR and negative towards HealthSpace

The decision whether to have an SCR involved balancing perceived benefits with perceived risks and was heavily influenced by previous personal experience (of illness, the healthcare system, government surveillance, or data loss) and the comprehension of some, "informed consent" might have unstable foundations - a finding which questions the adequacy of existing legislation about consent for the creation of, and access to, electronic patient records more generally. ${ }^{23}$

We thank the research participants for their time and honesty, and NHS staff in participating organisations for their cooperation and support. Yara Mohammad assisted with some of the interviews. The research was overseen by a multidisciplinary advisory group, which included patient representation

Contributors: TG conceptualised the study, obtained funding, assisted with interviews and focus groups, led the data analysis, wrote the paper with input from all coauthors, and is guarantor. GWW organised and ran all focus groups with assistance from other coauthors, including liaising with voluntary sector contacts, and took lead responsibility for ethical aspects of the research. TB, KS, and $\mathrm{SH}$ designed interview schedules and undertook interviews with individual patients. GWW, TB, and KS, along with a professional transcriber, undertook and checked the transcription of focus groups. All coauthors contributed to data analysis and the writing and revision of the paper

Funding: Department of Health through the Connecting for Health Evaluation Programme (CfHEP/002); the CFHEP is organised through Richard Lilford's team at the University of Birmingham. Competing interests: None declared.

Ethical approval: Thames Valley multicentre research ethics committee (06/MRE12/81 and subsequent amendments).

Provenance and peer review: Not commissioned; externally peer reviewed.

1 NHS Connecting for Health. Connecting for health business plan. London: Department of Health, 2004. www.connectingforhealth.nhs.uk

2 Brennan S. The NHS IT project. Oxford: Radcliffe, 2005.

3 National Audit Office. Department of Health: the national programme for IT in the NHS. Report by the comptroller and auditor general, HC 1173. London: Stationery Office, 2006.

4 Hillestad R, Bigelow J, Bower A, Girosi F, Meili R, Scoville R, et al. Can electronic medical record systems transform health care? Potential health benefits, savings, and costs. Health Aff (Millwood) 2005;24:1103-17.

5 Department of Health. Connecting for health: a guide to the national programme for information technology. London: DoH, 2005.

6 Anderson R. Confidentiality and connecting for health. Br J Gen Pract 2008;58:75-6.

7 Hanseth 0. Integration-complexity-risk: the making of information systems out-of-control. In: Ciborra CU, Hanseth O, eds. Risk, complexity and ICT. Oslo: Edward Elgar, 2007.

8 Keen J. The NHS programme for information technology: this massive natural experiment needs evaluating and regulating. $B M$ J 2006;333:3-4.

9 British Computer Society. The way forward for NHS health informatics: where should NHS connecting for health (NHS CFH) go from here? London: BCS,

2006. www.bcs.org/server.php?show=ConWebDoc.8951

10 Williams J. National programme for IT: the $£ 30$ billion question. $\mathrm{Br}$ J Gen Pract 2005;55:340-2.

11 Cayton H. Report of the ministerial taskforce on the NHS summary care record. London: Connecting for Health, 2006. www. connectingforhealth.nhs.uk/resources/care_record_taskforce_doc. pdf.

12 Greenhalgh T, Stramer K, Bratan T, Byrne E, Russell J, Mohammad Y, et al. Summary care record early adopter programme: an independent evaluation by University College London. London: University College London, 2008. www.ucl.ac.uk/openlearning/research.

13 Krippendorff K. Content analysis: an introduction to its methodology. Newbury Park, CA: Sage, 1980.

14 Ritchie J, Spencer L. Qualitative data analysis for applied policy research. In: Bryman A, Burgess RG, eds. Analysing qualitative data. London: Taylor and Francis, 1994.

15 Ross SE, Lin CT. The effects of promoting patient access to medical records: a review. J Am Med Inform Assoc 2003;10:129-38.

16 Gadd CS, Penrod LE. Assessing physician attitudes regarding use of an outpatient EMR: a longitudinal, multi-practice study. Proc AMIA Symp 2001:194-8.

17 Davies M, Eccles S, Braunold G, Winfield M, Thick M. Giving control to patients. BrJ Gen Pract 2008;58:148-9. 
18 Pyper C, Amery J, Watson M, Crook C. Patients' experiences when accessing their on-line electronic patient records in primary care. $\mathrm{Br}$ J Gen Pract 2004:54:38-43.

19 Adams T, Budden M, Hoare C, Sanderson H. Lessons from the central Hampshire electronic health record pilot project: issues of data protection and consent. BMJ 2004;328:871-4.

20 Wanless D. Securing the public health: taking a long term view. London: Department of Health, 2004.

21 Hibbard JH, Peters E, Dixon A, Tusler M. Consumer competencies and the use of comparative quality information: it isn't just about literacy. Med Care Res Rev 2007;64:379-94.
22 TNS UK. NHS connecting for health national and early adopter areas baseline report JN 133643/160085. London: TNS UK, 2007.

23 Montgomery J. Personal information in the National Health Service: the demise or rise of patient interests? In: Lace S, ed. The glass consumer: life in a surveillance society. London: National Consumer Council, 2005:187-204.

Accepted: 9 May 2008 